\title{
CARACTERIZACIÓN CLÍNICA, HISTOPATOLÓGICA E HISTOQUÍMICA DEL PAPILOMA CUTÁNEO EN BOVINOS (Bos taurus) DEL DEPARTAMENTO DE CÓRDOBA, COLOMBIA
}

\section{CLINICAL, HISTOPATHOLOGICAL AND HISTOCHEMICAL CHARACTERIZATION OF CUTANEOUS PAPILLOMATOSIS IN BOVINE (Bos taurus) OF CÓRDOBA DEPARTMENT, COLOMBIA}

\author{
José Cardona-Álvarez ${ }^{1}$, Donicer Montes-Vergara², Jaime Álvarez-Peñate ${ }^{3}$
}

\begin{abstract}
${ }^{1}$ MVZ, Esp, M.Sc, Ph.D., Profesor Titular de Medicina y Clínica de Grandes Animales, Grupo de Investigación en Medicina de Grandes Animales (MEGA), Facultad de Medicina Veterinaria y Zootecnia. Universidad de Córdoba - Montería, Colombia. Correspondencia: e-mail: jacardonaalvarez@correo.unicordoba.edu.co; ${ }^{2} Z$ Zootecnista, M.Sc, Ph.D., Departamento de Zootecnia, Facultad de Ciencias Agropecuarias. Universidad de Sucre - Sincelejo, Colombia, e-mail: donicer.montes@unisucre. edu.co; ${ }^{3}$ MVZ, Esp, MSc., Profesor Titular de Anatomía Veterinaria, Departamento de Ciencias Pecuarias. Universidad de Córdoba - Montería, Colombia, e-mail: jaimealvarezp@correo.unicordoba.edu.co.
\end{abstract}

Rev. U.D.C.A Act. \& Div. Cient. 21(1): 137-146, Enero-Junio, 2018

https://doi.org/10.31910/rudca.v21.n1.2018.672

\section{RESUMEN}

El objetivo de este trabajo fue estudiar los aspectos clínicos, histopatológicos e histoquímicos de la papilomatosis cutánea (PC) en bovinos (Bos taurus), del departamento de Córdoba, Colombia. Dicho estudio fue de tipo descriptivo, no probabilístico, en animales de conveniencia. Se utilizaron 24 bovinos con PCB, diagnosticados clínica e histopatológicamente. Las lesiones, se observaron a modo de masas exofíticas e hiperpigmentadas; en algunos casos, pediculadas o en forma de coliflor; en otros e inclusive en los papilomas planos, se evidenciaron los pedículos a la compresión. De igual forma, los papilomas de gran tamaño pendulantes fueron extraídos quirúrgicamente que, al ser diseccionados, se evidenció la hiperplasia epitelial, la hiperqueratosis y la proyección de la epidermis hacia la dermis. A nivel de cabeza, la mayor presentación se dio en boca, en orejas, en frente y en párpados. Histopatológicamente, en la coloración de H-E, se observó hiperplasia epitelial, acantosis, proyecciones digitales hacía la dermis e hiperqueratosis. En la coloración de T-G, se observó marcada proliferación dérmica de colágeno desorganizado, con escasa presencia de tejido conjuntivo difuso y en la coloración de $\mathrm{P}-\mathrm{R} / \mathrm{P}$, se observaron marcadas áreas de birrefringencia rojiza y escasa birrefringencia verdeamarillenta. El diagnóstico definitivo de la enfermedad, se fundamentó en las características clínicas, diagnóstico diferencial y en los hallazgos histopatológicos, siendo conclu- yentes, como métodos de diagnóstico de la PC en bovinos, del Departamento de Córdoba.

Palabras clave: Tumor, Papilomavirus, dermopatología, hiperqueratosis. Fuente: DeCs.

\section{SUMMARY}

The objective of this study was to study the clinical, histopathological and histochemical aspects of cutaneous papillomatosis (CP) in cattle (Bos taurus) of the department of Córdoba, Colombia. This study was descriptive, not probabilistic, in animals of convenience. Were used Twenty - four bovines with BCP diagnosed clinically and histopathologically. In describing the lesions exophytic and hyperpigmented masses were observed in some cases, pedicled or in the form of cauliflower in others and even in the flat papillomas showed the pedicles to the compression. Similarly, large pendulum papillomas were surgically removed, which when dissected revealed epithelial hyperplasia, hyperkeratosis and the projection of the epidermis to the dermis. At head level, the largest presentation was given in mouth, ears, forehead and eyelids. Histopathologically in the $\mathrm{H}-\mathrm{E}$ staining, were observed epithelial hyperplasia, acanthosis, digital projections to the dermis and hyperkeratosis. In the T-G staining, marked dermal proliferation of disorganized collagen with little diffuse connective tissue was present, and in the P-R / P 
staining, marked areas of reddish birefringence and scarce birefringence were observed. The definitive diagnosis of the disease was based on the clinical characteristics, differential diagnosis and histopathological findings, being conclusive as diagnostic methods for CP in bovines in the Department of Córdoba.

Key words: Tumor, Papillomavirus, dermopathology, hyperkeratosis. Source: DeCs.

\section{INTRODUCCIÓN}

La papilomatosis cutánea bovina (PCB) es una enfermedad infectocontagiosa y crónica, causada por virus de la familia Papillomaviridae, género Papillomavirus (BPV). Es un virus de distribución mundial, que presenta múltiples lesiones proliferativas de naturaleza fibroepitelial (Jelinek \& Tachezy, 2005), caracterizadas por la aparición de tumores benignos (fibropapilomas, papilomas o verrugas) en piel y en mucosas (Vivas et al. 2015). Las lesiones sufren regresión espontánea o progresan a la malignidad cuando son expuesto a co-factores ambientales (Módolo et al. 2017).

Los Papillomavirus pertenecen a una familia de virus que infectan gran variedad de animales, incluyendo bovinos (Silva et al. 2013). Los BPV son virus de ADN oncogénico bicatenarios, que suelen ser específicos de una especie, pero se pueden infectar de forma cruzada con otras especies, incluyendo búfalos, bisontes y équidos. Infecta las células epiteliales basales, que conducen a la formación de tumores, conocidos como papilomas o verrugas (Carvalho et al. 2012); estos virus en bovinos inducen lesiones benignas hiperplásicas de epitelios cutáneo y mucosal, pero en presencia de cofactores ambientales, estas lesiones también pueden desarrollar características neoplásicas (Módolo et al. 2017).

Comprenden catorce tipos, que han sido bien caracterizados y clasificados en 4 géneros: Deltapapillomavirus ( $\delta \mathrm{PV}$; BPV 1, 2, 13 у 14), Xipapillomavirus ( $\chi$ PV; BPV 3, 4, 6, 9, 11 y 12), Epsilonpapillomavirus (عPV; BPV 5 y 8); BPV 7 no se ha asignado, pero se ha propuesto para pertenecer al género Dyoxipapillomavirus (Roperto et al. 2016a). BPV 14 es un virus novedoso, cuyo genoma ha sido recientemente determinado (Munday et al. 2015).

De los genotipos virales caracterizados, hasta el momento, los BPV de 1 al 10 son estrictamente específicos de cada especie, pero los BPV 1 y 2 también puede infectar equinos, induciendo tumores fibroblásticos (Cardona et al. 2013e). Estas lesiones benignas, por lo general retroceden, pero también pueden persistir ocasionalmente, lo que conduce a un alto riesgo de evolucionar hacia el cáncer, en particular, en presencia de cofactores ambientales carcinógenos (Borzacchiello \& Roperto, 2008).
Se podría decir que, clínicamente, se puede sospechar del tipo de Papillomavirus presente en un paciente, según la ubicación de las lesiones; es así, como los Deltapapillomas se caracterizan por producir fibropapilomas cutáneos; los Xipapillomas, por producir lesiones epiteliales; los Epsilonpapillomas producen fibropapilomas y papilomas epiteliales (Catroxo et al. 2013), mientras que los Dyoxipapillomavirus presentan tropismo por células de diferentes órganos (Lunardi et al. 2016). Es así, como el BPV 1 y 2 se limita a la epidermis, ya que su ciclo de vida viral está estrechamente ligado a la diferenciación de queratinocitos de las capas espinosas y granulares (Brandt, 2016). El BPV 4, frecuentemente, se asocia con papilomas del canal digestivo superior; el BPV 5, 13 y 11, se han descrito en papilomas cutáneos y de pezón con características clínicas similares. Se sabe que los papilomas esofágicos ocurren en bovinos infectados con BVP 4, pudiendo experimentar progresión maligna en bovinos que se alimentan con helecho (Savini et al. 2016). De igual forma, los BPV 1, 2 y 13 también están asociados con la carcinogénesis de la vejiga en ganado adulto, que pastorea en tierras ricas en helechos (Roperto et al. 2016b).

La PCB es bastante común en bovinos jóvenes, sobre todo estabulados. La transmisión, se realiza por contacto directo con los animales afectados, a través de abrasiones cutáneas y por contacto indirecto, a través de fómites (instrumentos de tatuaje y descorne, cercas infectadas, narigueras, agujas hipodérmicas) (Vásqués et al. 2012; Catroxo et al. 2013); de igual forma, por las manos del ordeñador y hasta por la acción de vectores artrópodos, tales como garrapatas y tábanos, siendo la vía de ingreso del virus las pequeñas lesiones que se pueden generar en la piel del animal sano (Babaahmady \& Taherpour, 2011).

Módolo et al. (2017) manifiestan que la PCB ocasiona importantes pérdidas económicas en la ganadería bovina, debido al retraso en el desarrollo, la mala condición corporal y la reducción en la producción de leche. Asimismo, Catroxo et al. (2013) expresan pérdidas ocasionadas por el descarte prematuro de animales, mientras que Vásqués et al. (2012), explican que cuando los fibropapilomas se localizan en las pezuñas en el espacio interdigital, cojinetes y talones son dolorosos y pueden provocar desde cojeras hasta postración.

El diagnóstico, se basa en la caracterización clínica de las lesiones, la ubicación y la forma. Cardona et al. (2016) expresan que los papilomas presentan 3 características clínicas principales; son pediculadas (forma de coliflor), exofíticas e hiperqueratinizadas, mientras que Vivas et al. (2015) indican que los papilomas crecen lentamente en forma de nódulos, luego aceleran su crecimiento y se cornifican progresivamente, por lo que pueden llegar a ser de diferentes formas, pudiendo formar grandes masas de confluencia tumoral. Asimismo, Catroxo et al. (2013) explican que los papilomas 
más oscuros y bastante queratinizados se desprenden de la piel con mayor facilidad e informan que los nódulos, generalmente, se ulceran siendo invadidos por infecciones secundarias.

En la histopatología, se observa un crecimiento epitelial diferenciado con evidente hiperplasia, mostrando epidermis engrosada (acantosis), que emite proyecciones digitales hacia la dermis e hiperqueratosis, con tendencia al crecimiento y formación de túbulos de queratina; además de vacuolización nuclear en el estrato espinoso de la dermis (coilocitosis), con presencia de núcleos vacíos y otros cuerpos de inclusión (Iglesias \& Batista, 2003; Vásqués et al. 2012).

Entre los diagnósticos diferenciales es importante tener en cuenta diversas lesiones de la piel parecidas a papilomas, tales como lesiones por presencia de Dermatobia hominis (Cardona et al. 2013a), Demodex bovis (Cardona et al. 2013b), Pythium insidiosum (Cardona et al. 2013c) y carcinoma de células escamosas (Cardona et al. 2013d).

El objetivo de este trabajo fue realizar un estudio sobre los aspectos clínicos, histopatológicos e histoquímicos de la PC, en bovinos del departamento de Córdoba, Colombia.

\section{MATERIALES Y MÉTODOS}

Tipo de estudio. Se realizó un estudio de tipo descriptivo, no probabilístico en animales de conveniencia, con presencia de lesiones cutáneas compatibles con PCB.

Localización. Fue realizado en el departamento de Córdoba, Colombia, ubicado entre las coordenadas $7^{\circ} 23^{\prime}$ y $9^{\circ} 26^{\prime}$ LN y los $74^{\circ} 52^{\prime}$ y $76^{\circ} 32^{\prime}$ LO del meridiano de Greenwich, a una altura de $30 \mathrm{~m} \mathrm{~s}$. n. m, con temperatura promedio anual de $28^{\circ} \mathrm{C}$, humedad relativa del $82 \%$, precipitación media anual de $1400 \mathrm{~mm}$ y pertenece a la formación climática de bosque tropical lluvioso (Pabón et al. 2001).

Animales. Se utilizaron 24 bovinos, de diferentes razas y mestizajes, ambos sexos (16 machos y 8 hembras), con edades comprendidas entre 10 y 36 meses, que presentaron lesiones pediculadas (forma de coliflor), exofíticas e hiperqueratinizadas de la piel, compatibles con PC.

Los animales no fueron sometidos a dolor o estrés innecesario, por lo que fueron inmovilizados, teniendo en cuenta las normas técnicas en el manejo y la sujeción de animales, enmarcado en el cumplimiento de la Declaración Universal de los Derechos de los Animales, referente a los principios éticos internacionales para la investigación biomédica con animales del CIOMS (Council for International Organizations of Medical Sciences), establecida por la UNESCO (United Nations Educational, Scientific and Cultural Organization) y la OMS (Organización Mundial de la Salud) de 1949 y de la Ley 84 de Octubre 27 de 1989 (Estatuto Colombiano de Protección Animal) (Mrad, 2006).

A todos los animales, se les realizó evaluación clínica y caracterización anatomopatológica de las lesiones cutáneas, para lo cual, se tomaron fotografías de alta definición, para su posterior análisis (Sony DSC-HX10V, China). De igual forma, previa sedación (Xilacina $2 \%$, Bayer ${ }^{\circledR}$, Colombia) y aplicación de anestesia local (Lidocaína $2 \%$, Synthesis ${ }^{\circledR}$, Colombia), para realizar biopsias de tejido en el centro de la lesión tumoral, con punch de $6 \mathrm{~mm}$ (Monteiro et al. 2008), siendo fijadas en formalina al $10 \%$ por 24 horas, después colocadas en alcohol al $70 \%$ y llevadas al Laboratorio de Patología del Departamento de Ciencias Pecuarias de la Universidad de Córdoba, Colombia, donde fueron procesadas hasta su inclusión en parafina. Posteriormente, trasladadas al Laboratorio de Patología del Departamento de Veterinaria, de la Universidad Federal de Viçosa, Brasil, donde fueron procesadas para evaluación histopatológica e histoquímica, cortadas a $5 \mu \mathrm{m}$ de espesor, en un micrótomo (Leica RM2125 RTS®, Japón) y coloreadas con Hematoxilina - Eosina (H\&E), Tricrómico de Gomori (TG) y Picrosirius Red/Polarization (PR/P). Para el análisis foto microscópico de las muestras fue utilizado un microscopio con opción de polarización (Olympus BX-53®, Japón), del Laboratorio de Sistemática Molecular/BEAGLE del Departamento de Biología Animal, de la Universidad Federal de Viçosa, Brasil.

La presencia del papiloma, se determinó mediante caracterización clínica de la lesión por inspección y por palpación, en los lugares de común presentación, como región torácica, dorsal, cuello y cabeza y, en menor presentación, a nivel de miembros, periné, miembros, testículos y glándula mamaria. Fue utilizado el sistema de caracterización y de cuantificación de la PCB, de acuerdo con Monteiro et al. (2008), quienes sugirieron un sistema cualitativo de grados, de acuerdo con el porcentaje del cuerpo afectado por la presencia de papilomas, siendo leve $(<25 \%)$, moderado (entre el 25 y $50 \%)$ y grave $(>50 \%)$.

En la coloración de HE fue evaluada la presencia de hiperqueratosis, acantosis y la formación de papilas epidérmicas hacia la dermis. De igual forma en las coloraciones de TG y $\mathrm{PR} / \mathrm{P}$, se cuantificaron la presencia de fibras y tipo de colágeno.

Se utilizó la prueba de Chi cuadrado, con el fin de determinar diferencias significativas $(p \leq 0.05$,) entre las variables (clínica, histopatología e histoquímica). El análisis estadístico fue realizado con el auxilio del programa de computador S.A.S. 9.1.3 y para la tabulación y la organización de los datos, se utilizó el programa Microsoft Office Excel 2003. 


\section{RESULTADOS Y DISCUSIÓN}

Las manifestaciones clínicas observadas, las características anatomopatológicas de las lesiones y los resultados histopatológicos confirmaron el diagnóstico de PC en los 24 bovinos estudiados, de acuerdo con lo reportado, como método diagnóstico del PCB (Iglesias \& Batista, 2003; Vásqués et al. 2012; Catroxo et al. 2013; Vivas et al. 2015; Cardona et al. 2016).

En la tabla 1, se describe la distribución de los animales estudiados, según el sexo, edad, localización y graduación de las lesiones; el 66,7\% fueron machos y el 33,3\%, hembras. El $33,38 \%$ de los animales fueron menores de 12 meses; $54,2 \%$ estuvieron entre 12 y 24 meses y el $12,5 \%$, mayores de 24 meses. Respecto a la ubicación de las lesiones, el 41,7\% tuvo una ubicación a nivel de cabeza; el 25,0\%, de dorso; el $16,7 \%$, de miembros y el $12,5 \%$, a nivel de genitales. Importante resaltar que en el 50,0\% de los animales se presentaron lesiones en diferentes partes del cuerpo. De acuerdo con los grados, el $54,2 \%$ fueron graves; el $25,0 \%$, moderados y el $20,8 \%$, leves. No fueron observadas diferencias significativas en la presentación de papilomatosis $(\mathrm{P}>0,05)$ con respecto a la edad, el sexo y la ubicación de las lesiones en los animales del estudio.

Tabla 1. Distribución de las lesiones, según el sexo, la edad, la localización y la graduación, en 24 bovinos estudiados con PCB, en Córdoba, Colombia.

\begin{tabular}{|c|c|c|c|c|c|c|c|c|c|c|}
\hline \multirow{2}{*}{$\begin{array}{l}\text { SEXO } \\
N^{\circ}(\%)\end{array}$} & \multicolumn{3}{|c|}{ EDAD (Meses) $N^{\circ}(\%)$} & \multicolumn{4}{|c|}{ LOCALIZACIÓNIN (\%) } & \multicolumn{3}{|c|}{ GRADOS $N^{\circ}(\%)$} \\
\hline & $<12$ & 12 a 24 & $>24$ & Cabeza & Dorso & Miembros & Genitales & Leve & Moderado & Grave \\
\hline $\begin{array}{l}\text { Machos } \\
16(66,7)\end{array}$ & 5 & 9 & 2 & 7 & 4 & 3 & 2 & 3 & 4 & 9 \\
\hline $\begin{array}{c}\text { Hembra } \\
8(33,3)\end{array}$ & 3 & 4 & 1 & 3 & 2 & 2 & 1 & 2 & 2 & 4 \\
\hline TOTAL & $8(33,3)$ & $\begin{array}{c}13 \\
(54,2)\end{array}$ & $\begin{array}{c}3 \\
(12,5)\end{array}$ & $\begin{array}{c}10 \\
(41,7)\end{array}$ & $\begin{array}{c}6 \\
(25,0)\end{array}$ & $\begin{array}{c}4 \\
(16,7)\end{array}$ & $\begin{array}{c}3 \\
(12,5)\end{array}$ & $\begin{array}{c}5 \\
(20,8)\end{array}$ & $\begin{array}{c}6 \\
(25,0)\end{array}$ & $\begin{array}{c}13 \\
(54,2)\end{array}$ \\
\hline
\end{tabular}

Las manifestaciones clínicas de las lesiones cutáneas, se caracterizaron por la presencia de masas exofíticas e hiperpigmentadas; en algunos casos (Figura 1a), pediculadas o en forma de coliflor (Figura 1b); en otros e inclusive en los papilomas planos, se evidenciaron los pedículos a la compresión.
De igual forma, fue extraído quirúrgicamente un papiloma de gran tamaño pendulante (Figura 2a), hiperqueratinizado y pediculado (Figura $2 b$ ) que, al ser diseccionado, se evidencia la hiperplasia epitelial, la hiperqueratosis y la proyección de la epidermis hacia la dermis (Figura 2c).

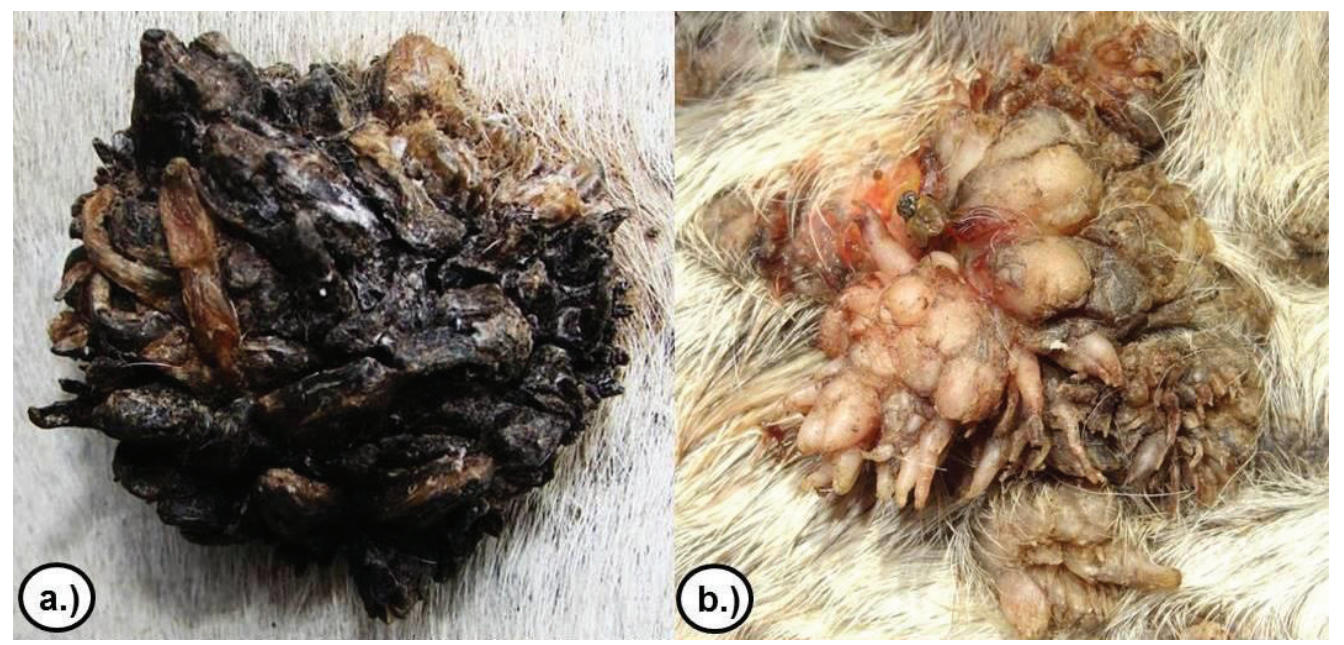

Figura 1. Caracterización clínica de la papilomatosis cutánea bovina. a.) Apariencia tumoral exofítica, de consistencia firme e hiperpigmentada y b.) Pediculadas o en forma de coliflor. 


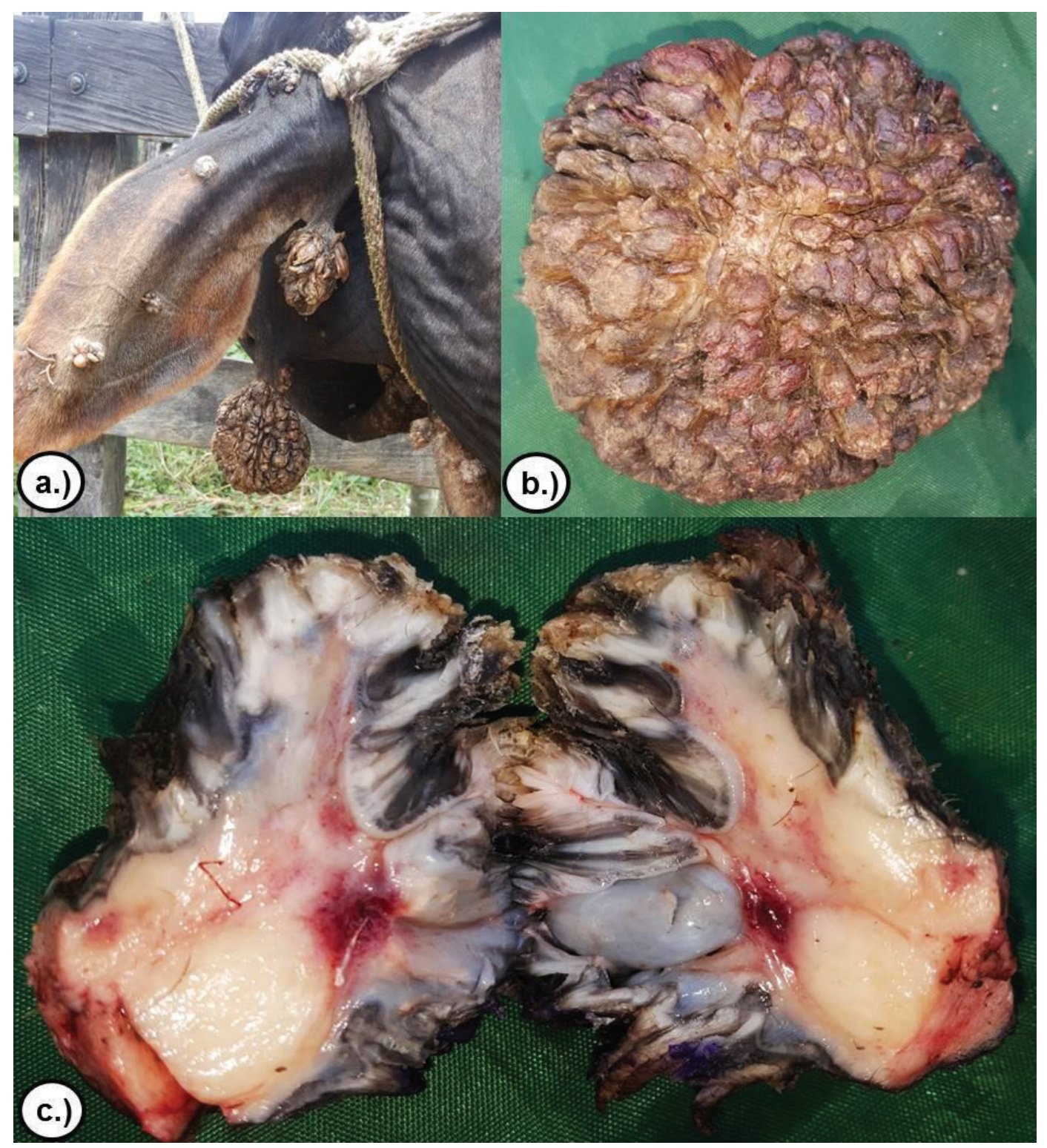

Figura 2. Extracción quirúrgica de un papiloma. a.) Papiloma de gran tamaño pendulante; b.) Hiperqueratinizado y pediculado y c.) Disección del papiloma con evidencia la hiperplasia epitelial, hiperqueratosis y la proyección de la epidermis hacia la dermis.

Las lesiones, se ubicaron en cabeza y en cuello (Figura 3a), en dorso (Figura 3b), así como en miembros y en genitales. A nivel de cabeza, los sitios de mayor presentación fueron en boca, en frente y párpados (Figura 4a), en orejas (Figura 4b), frente y párpados, respectivamente.

En todas las biopsias de tejido tumoral teñidas con la coloración de H-E, se observaron características histopatológicas similares entre ellas, las cuales, consistieron en la presencia de hiperplasia epitelial, acantosis, proyecciones digitales ha- cia la dermis e hiperqueratosis; de igual forma, se observó vacuolización nuclear en el estrato espinoso de la dermis (coilocitosis) (Figura 5a). En la coloración de T-G, se observó marcada proliferación dérmica de colágeno desorganizados, con escasa presencia de tejido conjuntivo difuso (Figura 5b) y en la coloración de $\mathrm{P}-\mathrm{R} / \mathrm{P}$, se observaron marcadas áreas de birrefringencia rojiza y escasa birrefringencia verde-amarillenta, lo que indica moderada presencia de colágeno maduro tipo I (color rojo brillante en la polarización) y escasa presencia de colágeno tipo III (color verde-amarillento brillante en la polarización) (Figura 5c y 5d). 


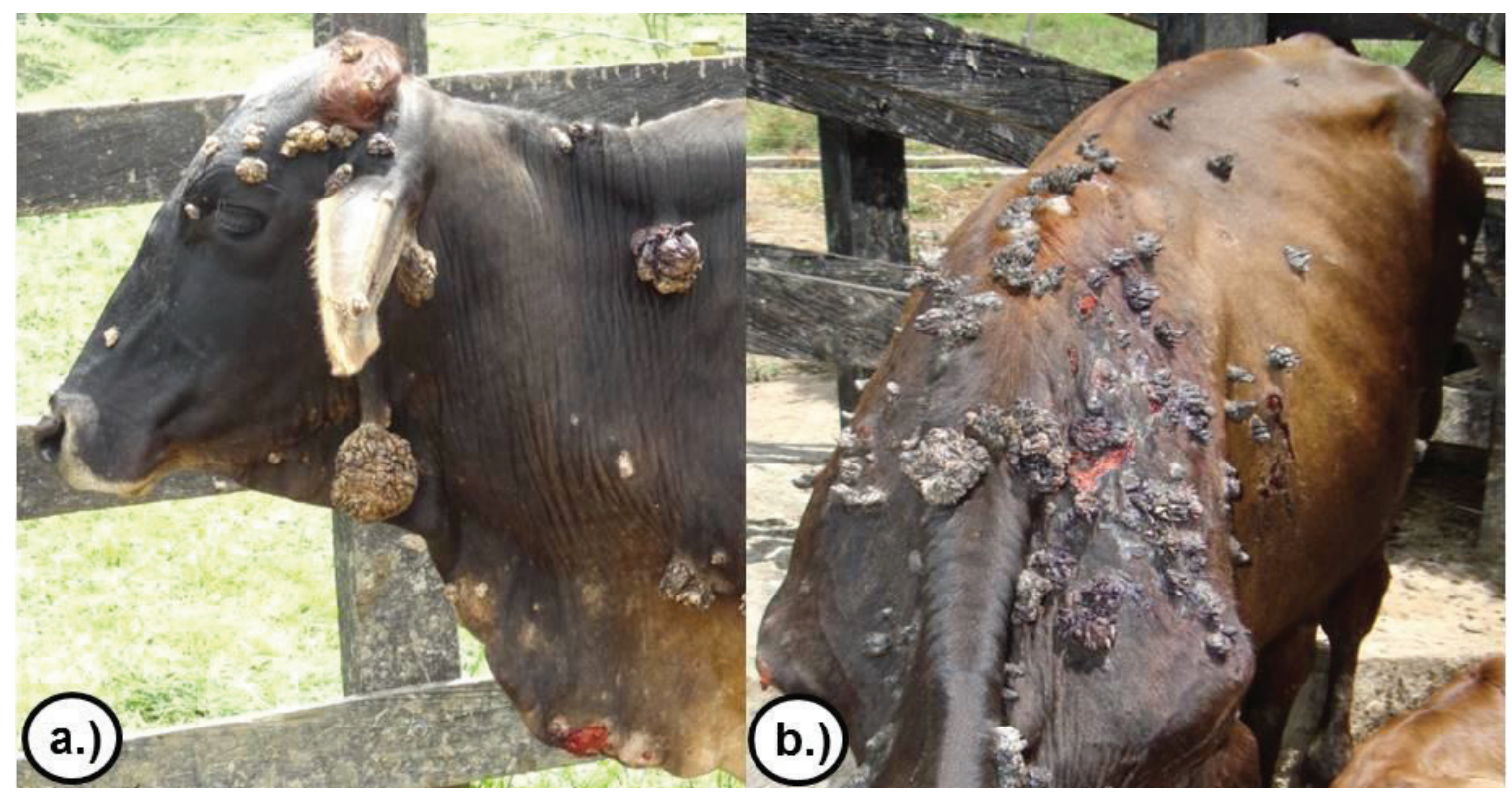

Figura 3. Ubicación de las lesiones en bovinos con papilomatosis cutánea. a.) Cabeza y cuello y b.) Dorso y tórax.

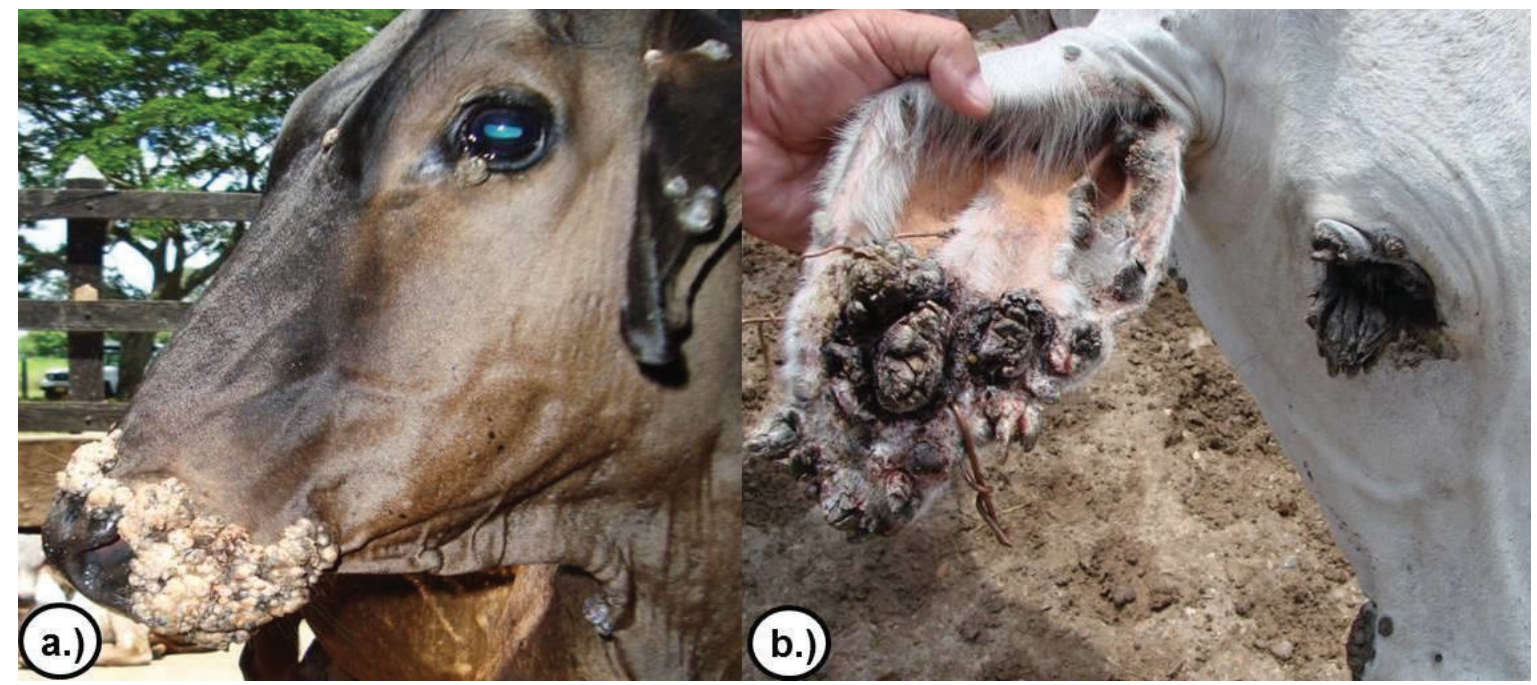

Figura 4. Ubicación de las lesiones a nivel de cabeza. a.) Boca, párpados y frente y b.) Orejas y párpados.

El PCB es una de las neoplasias dermatológicas más frecuentes, considerada la segunda neoplasia dermatológica de los bovinos, después del carcinoma de células escamosas, representando alrededor del 3\% de las neoplasias cutáneas en la especie (Reis et al. 2017); sin embargo, Cardona et al. (2017) y Buitrago et al. (2017) reportaron a la papilomatosis como la cuarta enfermedad dermatológica más frecuente en bovinos del departamento de Córdoba. De igual forma, Carvalho et al. (2014), la reportaron como la tercera causa neoplásica en rumiantes del nordeste brasilero, después del melanoma y del carcinoma de células escamosas. Catroxo et al. (2013) hallaron una prevalencia de papilomatosis bovina del $20 \%$ en Yucatán, México. Schuch (2007) manifiesta que, al ser los papilomas de fácil transmisión, son muy frecuentemente encontrados en la práctica clínica de campo, ya que por su apariencia macroscópica es muy bien conocida por los Médicos Veterinarios. 


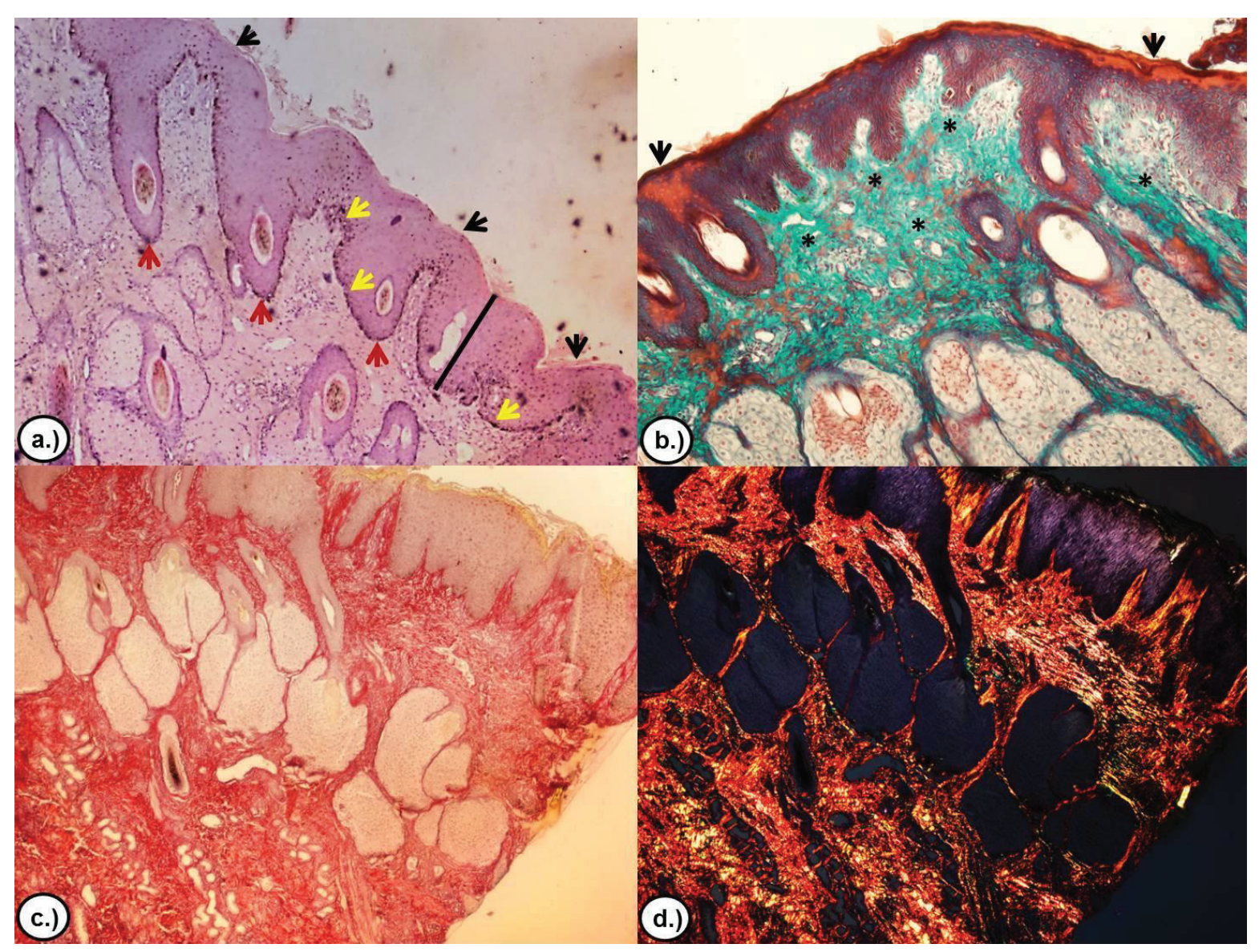

Figura 5. Análisis histopatológico del papiloma. a.) Tinción de H-E, se observa hiperplasia epitelial (barra), proyecciones digitales hacia la dermis (flechas rojas) e hiperqueratosis (flechas negras), así como vacuolización nuclear, en el estrato espinoso de la dermis (flechas amarillas) 10x; b.) Tinción de T-G, mostrando marcada proliferación dérmica de colágeno desorganizados, con escasa presencia de tejido conjuntivo difuso (asteriscos), así como la marcada hiperqueratosis (flechas) 10X; c.) y d.) Tinción de P-R/P sin y con polarización, respectivamente, mostrando marcadas áreas de birrefringencia rojiza y escasa birrefringencia verde-amarillenta, lo que indica moderada presencia de colágeno maduro tipo I y escasa presencia de colágeno inmaduro tipo III 10X.

La mayor presentación de PCB, se dio en animales jóvenes entre 12 y 24 meses de edad, coincidiendo con lo reportado por Vásqués et al. (2012) y Catroxo et al. (2013), indicando que, posiblemente, sea por el mayor contacto directo con los animales afectados, a través de abrasiones cutáneas y por contacto indirecto, por fómites. De igual forma, las lesiones cutáneas de los animales estudiados, se localizaron, primariamente, en cabeza, en dorso, en miembros y en genitales, contrario a lo reportado por García-Díaz (2017), quien registró mayor presencia de lesiones, a nivel de tórax, seguido de la cabeza. Monteiro et al. (2008) informaron mayor presentación en abdomen lateral, seguido de cabeza, así como lesiones mixtas; sin embargo, Carvalho et al. (2012) manifiestan que existe una marcada presencia de co-infecciones, encontrando más del $80 \%$ de las muestras analizadas de papilomas cutáneos en vacas, en Brasil, con infecciones de al menos 2 o 3 genotipos.

Las características macroscópicas de las lesiones de los animales del presente estudio fueron similares a las reportadas por Cardona et al. (2016); sin embargo, Vivas et al. (2015) indican que los papilomas crecen en forma de nódulos y se cornifican progresivamente, por lo que pueden llegar a ser de diferentes formas. Asimismo, Catroxo et al. (2013) explican que los papilomas más oscuros y bastante queratinizados se desprenden de la piel, siendo invadidos por infecciones secundarias. Con respecto a la graduación de las lesiones, en el presente estudio, la mayoría fueron graves, 
contrastando con lo informado por Monteiro et al. (2008), presumiblemente, por factores específicos, tales como la propia respuesta inmunológica del rebaño o a la cronicidad del proceso.

Los hallazgos histopatológicos con la coloración de H-E corresponden con los reportados en la literatura, para el diagnóstico del PCB, siendo las papilas epidérmicas, la hiperplasia epitelial y la acantosis el hallazgo más común (Iglesias \& Batista, 2003; Monteiro et al. 2008; Vásqués et al. 2012). De la misma forma, en la coloración de T-G, se evidenció moderada proliferación dérmica de colágeno; del mismo modo, en la coloración de P-R/P, se observaron pequeñas áreas de birrefringencia rojiza y verde amarillenta, indicando presencia moderada de colágeno maduro tipo I y tipo III; lo que podría indicar la buena respuesta regenerativa del organismo frente al proceso de progresión de células infectadas con Papillomavirus, siendo estas técnicas utilizadas como indicador del grado de respuesta de reparación en estados agresivos cutáneos (Cardona et al. 2013d; Cardona et al. 2016); no obstante, es preciso aclarar que no existen reportes del uso de técnicas histoquímicas, como T-G y P-R/P, como apoyo diagnóstico y pronóstico de la PC en bovinos.

En el aspecto económico es el segundo tumor cutáneo de mayor presentación en bovinos (Reis et al. 2017) y la cuarta patología más común en bovinos del departamento (Cardona et al. 2017; Buitrago et al. 2017), por lo que ocasiona pérdidas económicas importantes en la ganadería bovina, debido al retraso en el desarrollo, la mala condición corporal y la reducción en la producción de leche (Módolo et al. 2017), así como las ocasionadas por las cojeras y el descarte prematuro de animales (Vásqués et al. 2012; Catroxo et al. 2013).

Las características clínicas de las lesiones cutáneas analizadas concordaron con los hallazgos histopatológicos encontrados en 24 bovinos del departamento de Córdoba, con presencia de PC, siendo un aporte importante para el diagnóstico certero de la condición patológica, su diagnóstico clínico e histopatológico, por lo que se advierte sobre la presentación de la enfermedad en bovinos del departamento.

Agradecimientos: Al laboratorio de Patología del departamento de Ciencias Pecuarias de la Facultad de Medicina Veterinaria y Zootecnia, de la Universidad de Córdoba, Colombia; al laboratorio de Biología Estructural del departamento de Biología General y al laboratorio de Sistemática Molecular/BEAGLE del departamento de Biología Animal, de la Universidad Federal de Viçosa, Brasil, por su apoyo incondicional en el procesamiento y en el análisis de las muestras. Conflicto de intereses: El manuscrito fue preparado y revisado con la participación de todos los autores, quienes declaramos que no existe conflicto de intereses, que ponga en riesgo la validez de los resultados presentados.

\section{BIBLIOGRAFÍA}

1. BABAAHMADY, E.; TAHERPOUR, K. 2011. Verrugas en los pezones de vacas lecheras. Rev. Electrón. Vet. 12(6):1-6.

2. BORZACCHIELLO, G.; ROPERTO, F. 2008. Bovine papillomaviruses, papillomas and cancer in cattle. Rev. Vet. Res. 39(5):45.

3. BRANDT, S. 2016. Immune response to bovine papillomavirus type 1 in equine sarcoid. Rev. Vet. Journal. 216:107-108.

4. BUITRAGO-MEJIA, J.; DIAZ-CUETO, M.; SUAREZ-CHICA, A.; CARDONA-ALVAREZ, J. 2017. Distribución geográfica de la casuística clínica bovina del servicio ambulatorio de grandes animales de la Universidad de Córdoba (Colombia). Rev. Med. Vet. 34(Supl):101-113.

5. CARDONA, J.; MONTES, J.; CASTAÑO, F.; BLANCO, R.; GÓMEZ, V. 2013a. Frecuencia de dermatobiosis cutánea bovina en vacas Holstein de un hato lechero en Viçosa (MG, Brasil). Rev CES Med Zootec. 8(1):82-94.

6. CARDONA, J.; VARGAS, M.; PERDOMO, S. 2013b. Descripción clínica de la demodicosis bovina (Demodex bovis) en Córdoba, Colombia. Rev. Inv. Vet. Perú. 24(1):125-129.

7. CARDONA, J.; VARGAS, M.; GONZÁLEZ, M. 2013c. Evaluación clínica e histopatológica de la Pythiosis cutánea en terneros del departamento de Córdoba, Colombia. Rev. MVZ Córdoba. 18(2):3551-3558.

8. CARDONA, J.; VARGAS, M.; PERDOMO, S. 2013d. Estudio clínico e histopatológico del carcinoma de células escamosas de bovinos en el departamento de Córdoba, Colombia. Rev. Fac. Cs. Vets. UCV 54(2):68-77.

9. CARDONA, J.; VARGAS, M.; PERDOMO, S. 2013e. Estudio clínico e histopatológico del sarcoide fibroblástico en burros (Equus asinus) en Colombia. Revista Científica, FCV-LUZ XXIII (2):97-104.

10. CARDONA, J.; VARGAS, M.; PAREDES, E. 2016. Clinical and histopathological study of the phototoxic dermatitis in zebu calves in grazing of Brachiaria decumbens. Rev MVZ Córdoba. 21(2):5366-5380. 
11. CARDONA, J.; MARTÍNEZ, M.; MAZA, L. 2017. Casuística clínica más frecuente en el servicio ambulatorio de grandes animales de la Universidad de Córdoba, Colombia. Rev Col. Cienc Anim. 9(1):66-72.

12. CARVALHO, F.; DANTAS, A.; RIET-CORREA, F.; ANDRADE, R.; NÓBREGA, P.; MIRANDA, E.; SIMÕES, S.; AZEVEDO, S. 2014. Estudo retrospectivo das neoplasias em ruminantes e equídeos no semiárido do Nordeste Brasileiro. Pesq. Vet. Bras. 34(3):211-216.

13. CARVALHO, F.; DANTAS, A.; RIET-CORREA, F.; MIRANDA-NETO, E.; SIMÕES, S.; AZEVEDO, S. 2012. Fatores de risco associados à ocorrência de carcinoma de células escamosas em ruminantes e equinos no semiárido da Paraíba. Pesq. Vet. Bras. 32(9):881886.

14. CATROXO, M.; MARTINS, A.; PETRELLA, S.; SOUZA, F.; NASTARI, B. 2013. Ultrastructural Study of Bovine Papillomavirus During Outbreaks in Brazil. International J. Morph. 31(2):777-784.

15. GARCÍA-DÍAZ, J. 2017. Efecto de la suplementación parenteral de cobre Zinc y Manganeso en el tratamiento de la papilomatosis cutánea bovina. REDVET. 18(1):110.

16. IGLESIAS, A.; BATISTA, L. 2003. Caracterização anatomopatológica da papilomatose cutânea em bovinos leiteiros. Rev. Bras. Ciênc. Vet. 10(3):161-165.

17. JELINEK, F; TACHEZY R. 2005. Cutaneous papilomatosis in cattle. Rev. J. Comp. Pathol. 132:70-81.

18. LUINARDI, M.; DE CAMARGO, C.; ALFIERI, A.; DE ALCÂNTARA, B.; VILAS, L.; OTONEL, R. 2016. Genetic diversity of bovine papillomavirus types, including two putative new types, in teat warts from dairy cattle herds. Arch. Virology. 161(6):1569-1577.

19. MÓDOLO, D.; PINHEIRO, R.; MAZZUCHELLI, J.; PERERIRA, A.; CARVALHO, D.; ZANPHORLIN, L.; BEÇAK, W.; MENOSSI, M.; CASSIA, R.; FRANCO, R. 2017. Integrated analysis of recombinant BPV-1 L1 protein for the production of a bovine Papillomavirus VLP vaccine. Rev Vaccine. 35:15901593.

20. MONTEIRO, V.; CARDOSO-COELHO, M.; CARNEIRO, A.; SILVA, R.; TEIXEIRA, M.; WANDERLEY, A.; WANDERLEY, E.; FRANCO, E. 2008. Descrição clínica e histopatológica da papilomatose cutânea bovina (bpv). Ciênc. Anim. Bras. 9(4):1079-1088.
21. MRAD, A. 2006. Ética en la investigación con modelos animales experimentales. Alternativas y las 3 RS de Russel. Una responsabilidad y un compromiso ético que nos compete a todos. Rev. Col. Bioética. 1(1):163-184.

22. MUNDAY, J.; THOMSON, N.; DUNOWSKA, M.; KNIGHT, C.; LAURIE, R.; HILLS, S. 2015. Genomic characterization of the feline sarcoid-associated papillomavirus and proposed classification as Bos taurus papillomavirus type 14. Rev Vet. Microbiol. 177(34):289-295.

23. PABÓN, J.; ESLAVA, J.; GÓMEZ, R. 2001. Generalidades de la distribución espacial y temporal de la temperatura del aire y de la precipitación en Colombia. Meteorol. Colomb. 4:47-59.

24. REIS, M.; SLAVIERO, M.; LORENZETT, M.; CRUZ, R.; GUIMARÃES, L.; PAVARINI, S.; DRIEMEIER, D.; SONNE, L. 2017. Neoplasmas bovinos diagnosticados no Setor de Patologia Veterinária da UFRGS, Porto Alegre (2005-2014). Pesquisa Vet. Bras. 37(2):105-109.

25. ROPERTO, S.; MUNDAY, J.; CORRADO, F.; GORIA, M.; ROPERTO, F. 2016a. Detection of bovine papillomavirus type 14 DNA sequences in urinary bladder tumors in cattle. Rev. Vet. Microbiol. 190:1-4.

26. ROPERTO, S.; RUSSO, V.; LEONARDI, L.; MARTANO, M.; CORRADO, F.; RICCARDI, M.; ROPERTO, F. 2016b. Bovine papillomavirus type 13 expression in the urothelial bladder tumours of cattle. Rev. Transbound. Emerg. Dis. 63(6):628-634.

27. SAVINI, F.; MANCINI, S.; GALLINA, L.; DONATI, G.; CASÁ, G.; PELI, A.; SCAGLIARINI, A. 2016. Bovine papillomatosis: First detection of bovine papillomavirus types 6, 7, 8, 10 and 12 in Italian cattle herds. Rev. Vet Journal. 210:82-84.

28. SCHUCH, L. 2007. Papilomatose bovina. In: RIET-CORREA, F.; SCHILD, A.; LEMOS, R.; BORGES, J. (Eds), Doenças de Ruminantes e Equídeos. Vol.1. Pallotti, Santa Maria, Brasil. p.179-182.

29. SILVA, M.; ALTAMURA, G.; CORTEGGIO, A.; ROPERTO, F.; BOCANETI, F.; VELESCU, E.; FREITAS, A.; CARVALHO, C.; CAVALCANTI, K.; BORZACCHIELLO, G. 2013. Expression of connexin 26 and bovine papillomavirus E5 in cutaneous fibropapillomas of cattle. Ver. Vet. Journal. 195(3):337-343. 
30. VÁSQUÉS, R.; ESCUDERO, C.; DOMÉNECH, A.; GÓMEZ, E.; BENÍTEZ, L. 2012. Papilomatosis bovina: epidemiología y diversidad de papilomavirus bovinos (BPV). Rev. Complut. Cienc. Vet. 6(2):38-57.
31. VIVAS, R.; CORDERO, L.; RUIZ, E.; CÁRDENAS, M.; MELINA, M.; NAVARRO, R. 2015. Papilomatosis bovina en el trópico mexicano: presentación clínica y control. Bioagrociencias. 8(1):45-52.

Recibido: Septiembre 21 de 2017

Aceptado: Febrero 1 de 2018

Cómo citar:

Cardona-Álvarez, J.; Montes-Vergara, D.; Álvarez-Peñate, J. 2018. Caracterización clínica, histopatológica e histoquímica del papiloma cutáneo en bovinos (Bos taurus) del departamento de Córdoba, Colombia. Rev. U.D.C.A Act. \& Div. Cient. 21(2): 137-146. 